\title{
STAR FORMATION AND INFRARED EMISSION IN GALAXIES
}

\author{
Nikolaos D. Kylafis and Angelos Misiriotis \\ kylafis@physics.uoc.gr \\ University of Crete \\ Department of Physics \\ P.O. Box 2208 \\ 71003 Heraklion, Crete \\ GREECE
}

\begin{abstract}
The relationship between star formation and infrared emission in galaxies will be investigated. If galaxies were simple objects and young stars were completely covered with dust, then all the absorbed light of the young stars would be reemitted in the infrared and from the infrared emission of galaxies we would infer the star formation rate (SFR) in them accurately. To show the complexities involved in real galaxies, we will use as a case study the late-type spiral galaxies. We will show that the heating of the dust is done mainly by the UV radiation of the young stars and therefore the infrared emission reveals the SFR in them. With a realistic model and its application to a number of galaxies, tight correlations are derived between SFR and total far infrared luminosity on one hand, and dust mass and 850 micron flux on the other. Other diagnostics of the SFR are examined and it is shown that there is consistency among them. Thus, the SFR for galaxies of all Hubble types has been determined as well as for interacting starburst galaxies. Combining different methods, the star-formation history of the universe has been determined and will be shown. Finally, some early results from the Spitzer Space Telescope will be presented.
\end{abstract}

Keywords: Star formation, infrared emission, galaxies

\section{Introduction}

We will start by asking the naive and rhetoric question of why should star formation and infrared emission in galaxies be connected.

If galaxies were simple objects, consisting of young stars surrounded by optically thick dust, then practically all the luminosity of the young stars would be absorbed by the dust and it would be re-emitted in the infrared (IR) part of the spectrum. Then, observation of the IR luminosity of a galaxy would give 
us, with the use of an initial mass function, the star formation rate (SFR) in the galaxy.

However, real galaxies are more complicated objects for the following reasons: 1) They consist mainly of old stars, which may also contribute to the heating of the dust. 2) The dust is distributed throughout a galaxy and not around its stars. 3) The optical depth of the dust in a galaxy varies significantly with position in the galaxy and direction. Therefore, detailed modeling of the stars (young and old) and the dust in a galaxy is needed before trustworthy conclusions are drawn.

In what follows, we will treat late-type spiral galaxies as a case study. Then we will extend our discussion to all types of galaxies.

\section{Model for late-type spiral galaxies}

\section{Modeling of the optical light}

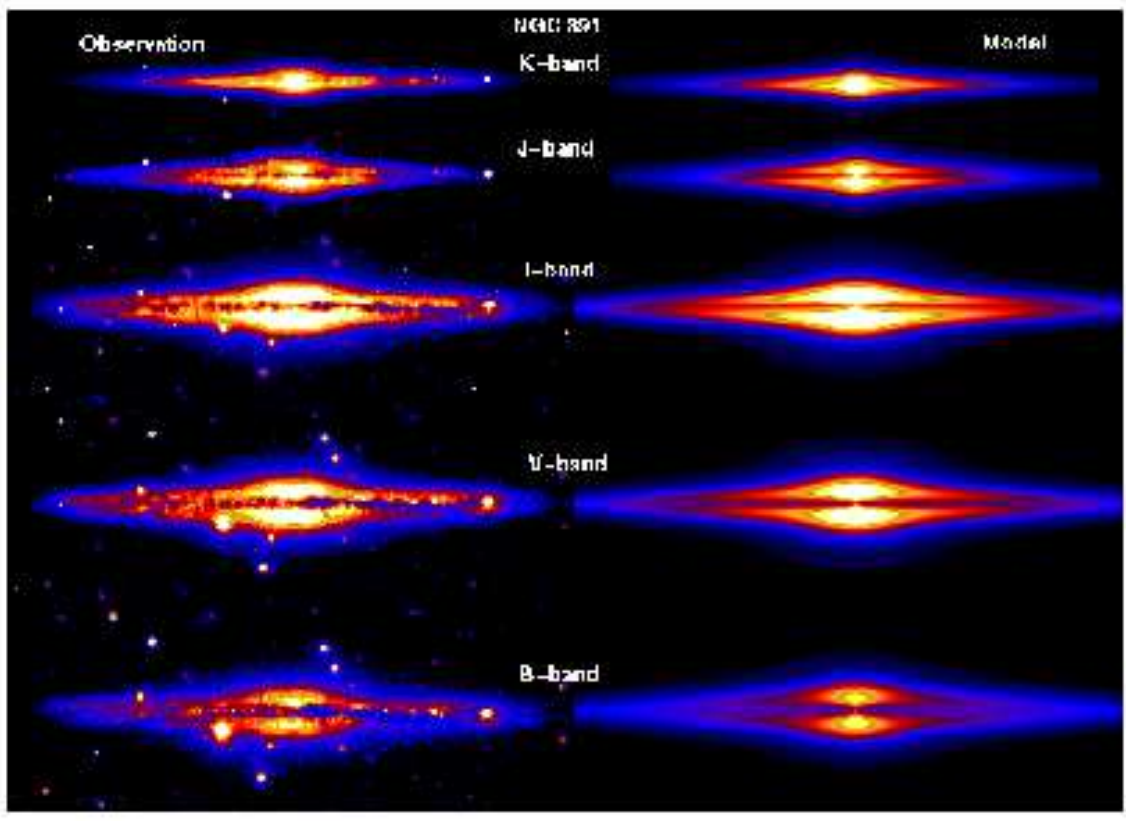

Figure 1. Images of NGC 891 in the K, J, I, V, and B bands and the corresponding models.

A successful model for late-type spiral galaxies has been applied to a number of galaxies by Xilouris et al. (1999). This model includes the old stellar 

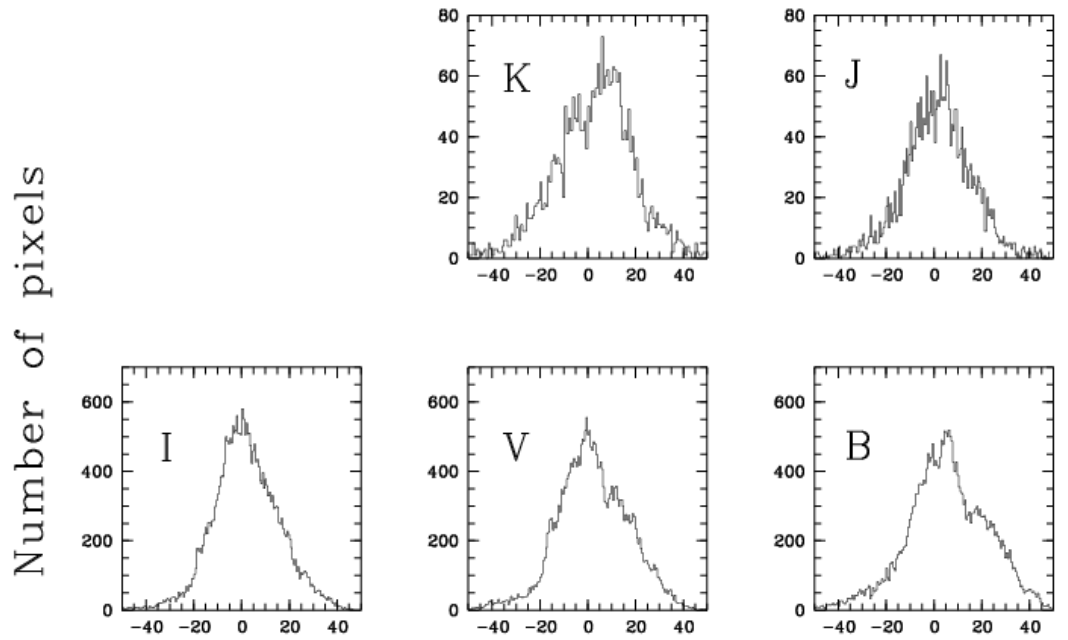

Relative error \%

Figure 2. Histograms of the relative errors between the pixels of the K, J, I, V, and B band observations and the corresponding models.

population in the form of an exponential (both in radius and height) disk and a de Vaucouleurs (1953) spheroid.

The dust is distributed in another exponential disk (with scales different than those of the stars). Comparison of model images with optical and near infrared (NIR) images of galaxies reveals the total amount of dust (warm and cold) in them and its distribution. Figure 1 shows NGC 891 in five bands and the corresponding model images (Xilouris et al. 1998). The agreement between the model and the observations is very good. This is seen more clearly in Figure 2, where the residuals between the model and the observations are shown (Xilouris et al. 1998). The main conclusions of Xilouris et al. (1999) are:

1) The scale height of the dust is about half that of the stars.

2) The radial scale length of the dust is about 1.4 times that of the stars. Furthermore, the dust extends beyond the optical disk.

3) The average gas-to-dust ratio of seven spiral galaxies is about 400 , i.e. comparable to that of our Galaxy. 
4) The extinction coefficient in the optical and NIR parts of the spectrum is the same as in our Galaxy, indicating common dust properties among spiral galaxies.

5) The central, face-on optical depth in the B-band of all seven galaxies is less than one. This means that, if all the dust has been accounted for in these galaxies, then late-type spiral galaxies are transparent.

Similar conclusions have been reached by Alton et al. (1998) and Davies et al. (1999). The effects of spiral structure have been shown to be negligible (Misiriotis et al. 2000) and similarly for the effects of clumpiness in the dust (Misiriotis and Bianchi 2002).

\section{Modeling of the infrared emission}
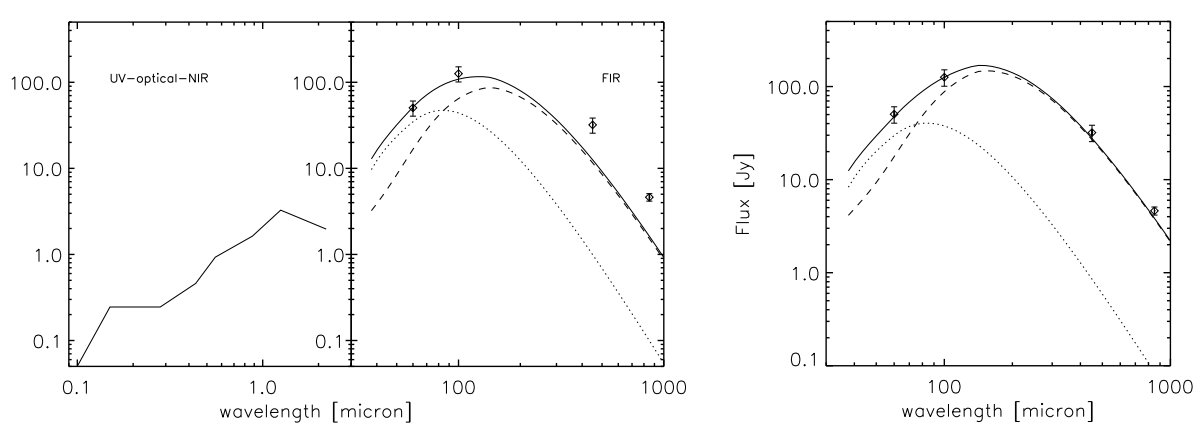

Figure 3. One- and two-dust-disk models of NGC 891.

In order to model the IR emission of late-type spiral galaxies, one has to take into account the heating of the dust not only by the old stars but also by the young ones. This was done by Popescu et al. (2000) for NGC 891 and by Misiriotis et al. (2001) for four more galaxies. The model utilizes the dust distribution derived from the optical images and assumes that the young stars (and therefore the UV emission) are distributed in an exponential disk with a small scale height $(90 \mathrm{pc}$ ) and scale length equal to the B-band scale length of the old stellar population. Part of the UV luminosity is absorbed by local sources (HII complexes) and the rest is diffuse. The far infrared (FIR) emissivity of the dust was taken to be the same as the one thought appropriate for our Galaxy (Laor \& Draine 1993).

It was found that model fluxes in the submillimeter (submm) part of the spectrum are significantly lower than the observed ones (Fig. 3, left). In order to account for the "missing" submm flux, Popescu et al. (2000) proposed that there is a second dust disk, not visible in the optical images, with scale height 
$90 \mathrm{pc}$ (equal to that of the young stars). The mass in the second dust disk is about the same as that of the first. The model accounts (Fig. 3, right) not only for the spectral energy distribution (SED) of NGC 891 in the FIR/submm regime (Popescu et al. 2000), but also for its observed surface brightness at 170 and $200 \mu \mathrm{m}$ (Popescu et al. 2004). Furthermore, the model showed that the heating of the dust is done mainly by the UV. Thus, the star formation rate in NGC 891 is directly connected to its infrared emission.

It is, however, possible that the FIR/submm dust emissivity value used for our Galaxy (e.g. Draine \& Lee 1984) has been underestimated. Alton et al. (2004), using a simple but reliable model, studied the edge-on galaxies NGC 891, NGC 4013, and NGC 5907 and concluded that the emissivity at $850 \mu \mathrm{m}$ is about four times the widely adopted value of Draine \& Lee (1984). A detailed model by Dasyra et al. (2004) arrived at the same conclusion.

At the moment, the above degeneracy (i.e., more dust or higher emissivity) is not lifted, but the higher emissivity seems to be a real possibility (Dasyra et al. 2004).

\section{Correlation between star formation and infrared emission}

As we saw above, in order for one to fit the SED of a late-type spiral galaxy, a SFR must be assumed. The question then is: Can a correlation be found between SFR and infrared emission for a sample of late-type spiral galaxies?

The answer is yes and it was given by Misiriotis et al. (2004). They studied 62 bright IRAS galaxies from the SCUBA Local Universe Galaxy Survey of Dunne et al. (2000). Figure 4 shows the derived dust mass of the galaxies as a function of their $850 \mu \mathrm{m}$ luminosity. The correlation is linear and quite impressive. This is not surprising, because most of the dust in these galaxies (as in the galaxies studied by Xilouris et al. 1999) is cold ( $\sim 15 \mathrm{~K})$. In Figure 5, the derived SFR is shown as a function of the $100 \mu \mathrm{m}$ luminosity. Since the peak of the SED of these galaxies is near $100 \mu \mathrm{m}$, it is again not surprising that the correlation is linear and quite tight. In Figure 6, the sought after correlation between SFR and IR luminosity is shown. The solid line is a linear fit to the data, while the dashed one is the correlation of Kennicutt (1998; see also Buat \& Xu 1996). The dot-dashed line shows the Kennicutt correlation if one assumes that the UV to IR transformation efficiency is not $100 \%$ (as in starbursts) but $50 \%$.

\section{Other star formation diagnostics}

Several other SFR diagnostics have been used over the years and the question is how well they agree among themselves. The answer now is quite well, though this was not the case a few years ago. 


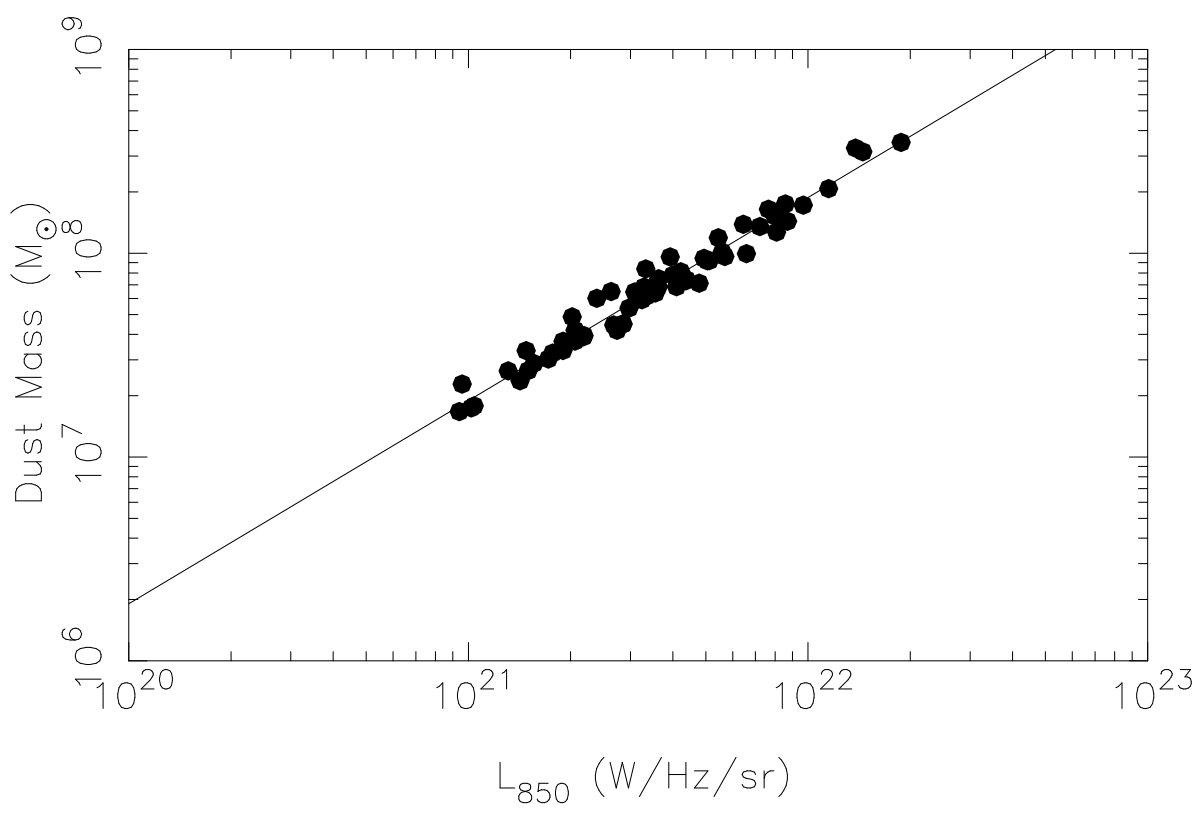

Figure 4. Dust mass as a function of luminosity at $850 \mu \mathrm{m}$. The solid line shows the best linear fit (in log-log space).

Kewley et al. (2002) showed that the SFR derived from the IR luminosity and that derived from $\mathrm{H} \alpha$ (properly corrected for extinction) are in excellent agreement for all types of galaxies (from elliptical, to spiral, to peculiar) and for SFR ranging over four orders of magnitude. The reader is also referred to the work of Buat et al. (2002), Hirashita et al. (2003), Panuzzo et al. (2003), and Flores et al. (2004).

The forbidden lines of [OII] have also been used as a measure of SFR. Kewley et al. (2004) showed that the SFR derived from [OII] and that derived from $\mathrm{H} \alpha$ are in excellent agreement for all types of galaxies and for four orders of magnitude of the SFR.

Not surprisingly, the SFR determined from UV observations is in excellent agreement with the SFR determined from the IR (Iglesias-Paramo et al. 2004). On the other hand, what is somewhat surprising is the fact that the $1.4 \mathrm{GHz}$ luminosity is a good indicator of the SFR. For an explanation of this, the reader is referred to Bressan et al. (2002), but the explanation is not widely accepted (Bell 2003; Pierini et al. 2003). Afonso et al. (2003) showed that the SFR determined from the $1.4 \mathrm{GHz}$ luminosity is in good agreement with the SFR determined from $\mathrm{H} \alpha$ or [OII]. 


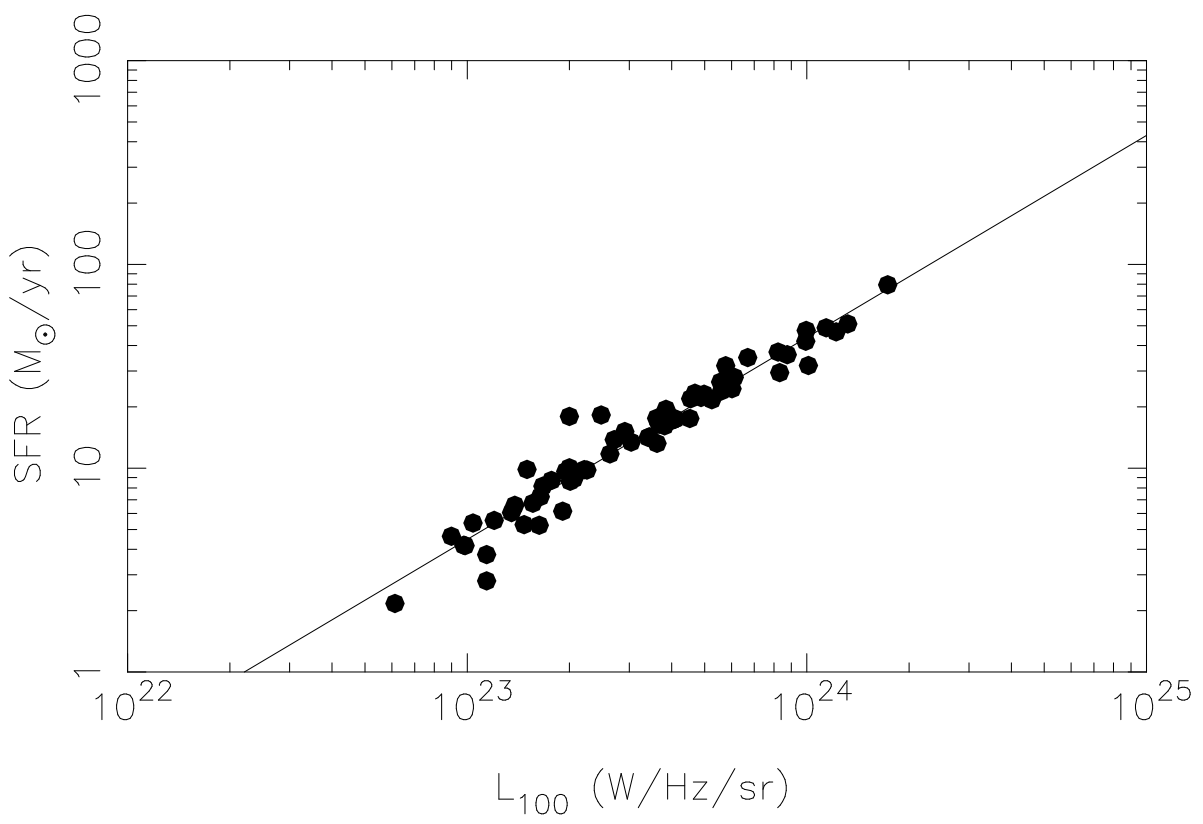

Figure 5. Star formation rate plotted as a function of $L_{100}$. The solid line is the best linear fit (in log-log space).

It is remarkable that even for interacting starburst galaxies there is a good correlation between the SFR derived from the $\mathrm{H} \alpha$ flux and the SFR derived from the FIR continuum (Dopita et al. 2002).

Last but not least, we want to mention the work of Ferster Schreiber et al. ( 2004), who used a sample of galaxies containing a) quiescent spiral galaxies, b) spiral galaxies with active circumnuclear regions, c) starburst galaxies, d) LIRGs, and e) ULIRGs and showed that the monochromatic $15 \mu \mathrm{m}$ continuum and the $5-8.5 \mu \mathrm{m}$ emission constitute excellent indicators of SFR.

We believe that the above are more than convincing that the determination of the SFR in galaxies is a mature subject. Significant progress has also been made in the determination of the SFR history. The most recent work in this subject is that of Heavens et al. (2004), who did an analysis of the 'fossil record' of the current stellar populations of 96,545 nearby galaxies, from which they obtained a complete star-formation history. They found that the peak of star formation was at about five billion years ago, i.e. more recently than other studies had found. They also found that the bigger the stellar mass of the galaxy, the earlier the stars were formed, which indicates that high- and lowmass galaxies have very different histories. 


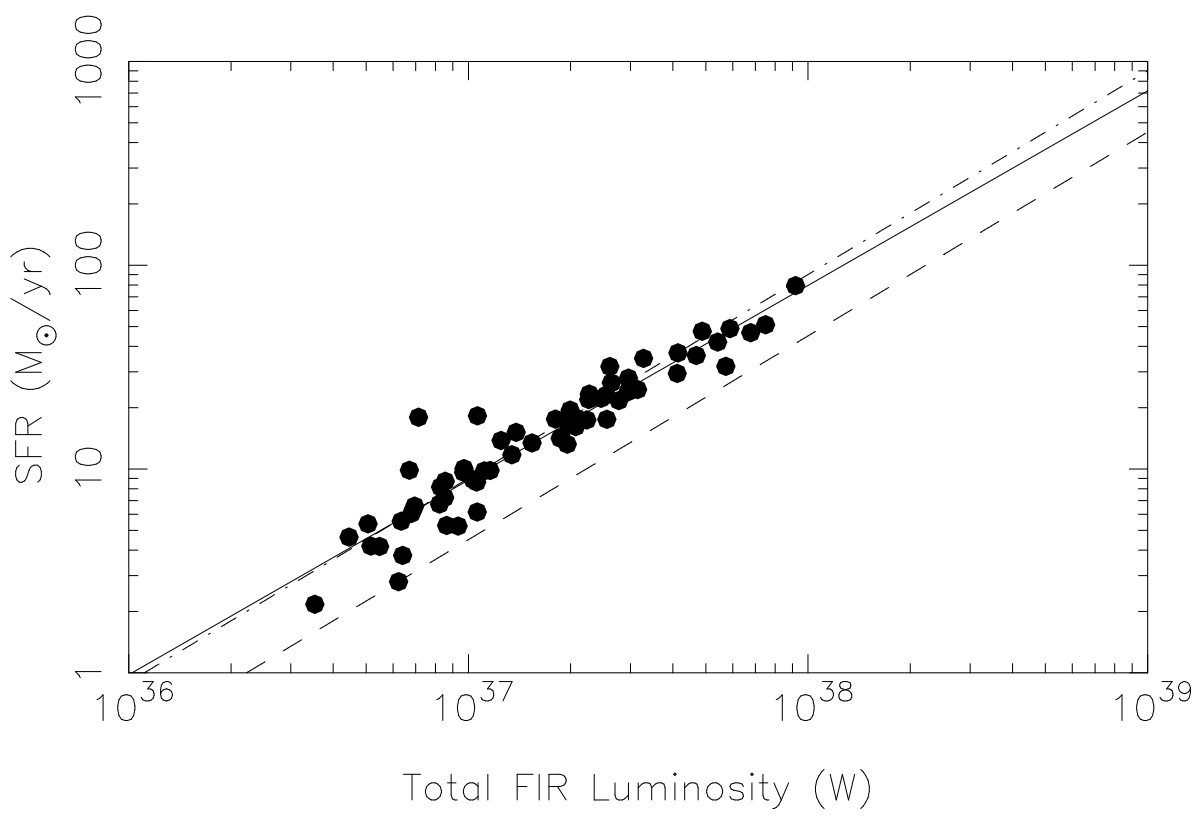

Figure 6. Star formation rate as a function of total FIR luminosity. The solid line is the best linear fit (in log-log space). The dashed line is drawn according to the SFR- $L_{F I R}$ relation of Kennicutt (1998) for starburst galaxies. The dash-dotted line is Kennicut's relation if one 50\% rather than $100 \%$ transformation efficiency of UV into FIR

\section{Instead of a summary}

Instead of a summary, we want to mention just three of the recently reported very exciting results from the Spitzer Space Telescope.

Appleton et al. (2004) reported that the FIR - radio correlation is valid to at least $z=1$ and similarly for the mid-infrared - radio correlation.

Higdon et al. (2004; see also Charmandaris et al. 2004) reported that the redshift of high- $z$, faint galaxies can be determined from the $14-38 \mu \mathrm{m}$ spectrum! This opens up tremendous possibilities for the determination of $z$ of faint galaxies.

Houck et al. (2004) reported that the blue compact dwarf galaxy SBS 0335052 , which has very low metallicity $\left(Z \sim Z_{\odot} / 41\right)$, has a featureless midinfrared spectrum with a peak at $\sim 28 \mu \mathrm{m}$ ! Taken at face value, this means that there is no cold dust in this galaxy.

The reader is referred to the Special Issue of ApJS (Volume 154) for additional exciting results from Spitzer. 


\section{References}

Afonso, J., Hopkins, A., Mobasher, B., Almeida, C. 2003, ApJ, 597, 269

Alton, P. B., Trewhella, M., Davies, J. I., et al. 1998, A\&A, 507, 125

Alton, P. B., Xilouris, E. M., Misiriotis, A., Dasyra, K. M., Dumke, M. 2004, A\&A, 425, 109

Appleton, P. N., Fadda, D. T., Marleau, F. R., et al. 2004, ApJS, 154, 147

Bell, E. F. 2003, ApJ, 586, 794

Bressan, A., Silva, L., Granato, G. L. 2002, A\&A, 392, 377

Buat, V., Boselli, A., Gavazzi, G., Bonfanti, C., 2002, A\&A, 383, 801

Buat, V., Xu, C. 1996, A\&A, 306, 61

Charmandaris, V.; Uchida, K. I.; Weedman, D., et al., 2004, ApJS, 154, 142

Dasyra, K. M., Xilouris, E. M., Misiriotis, A., Kylafis, N. D. 2004,A\&A submitted

Davies, J. I., Alton, P., Trewhella, M., Evans, R., Bianchi, S. 1999, MNRAS, 304, 495

de Vaucouleurs, G. 1953, MNRAS, 113, 134

Dopita, M. A., Pereira, M., Kewley, L. J., Capaccioli, M. 2002, ApJS, 143, 47

Draine, B. T., Lee, H. M. 1984, ApJ, 285, 89

Dunne, L., Eales, S., Edmunds, M., Ivison, R., Alexander, P., Clements, D. L. 2000, MNRAS, 315,115

Flores, H., Hammer, F., Elbaz, D., et al. 2004, A\&A, 415, 885

Ferster Schreiber, N. M., Roussel, H., Sauvage, M., Charmandaris, V. 2004, A\&A, 419, 501

Heavens, A., Panter, B., Jimenez, R., Dunlop, J. 2004, Natur, 428, 625

Higdon, S. J. U., Weedman, D., Higdon, J. L., et al. 2004, ApJS, 154, 174

Hirashita, H., Buat, V., Inoue, A. K. 2003, A\&A, 410, 83

Houck, J. R., Charmandaris, V., Brandl, B. R. 2004, ApJS, 154, 211

Iglesias-Paramo, J., Buat, V., Donas, J., Boselli, A., Milliard, B. 2004, A\&A, 419, 109

Kennicutt, R. C., Jr. 1998, ARA\&A, 36, 189

Kewley, L. J., Geller, M. J., Jansen, R. A., Dopita, M. A. 2002AJ, 124, 3135

Kewley, L. J., Geller, M. J., Jansen, R. A. 2004, AJ, 127, 2002

Laor, A., Draine, B. T. 1993, ApJ, 402, 441

Misiriotis, A., Bianchi, S. 2002, A\&A, 384, 866

Misiriotis, A., Kylafis, N. D., Papamastorakis, J., Xilouris, E. M. 2000, A\&A, 353, 117

Misiriotis, A., Papadakis, I. E., Kylafis, N. D., Papamastorakis, J. 2004, A\&A, 417, 39

Misiriotis, A., Popescu, C. C., Tuffs, R., Kylafis, N. D. 2001, A\&A, 372, 775

Panuzzo, P., Bressan, A., Granato, G. L., Silva, L., Danese, L. 2003, A\&A, 409, 99

Pierini, D., Popescu, C. C., Tuffs, R. J., Velk, H. J. 2003, A\&A, 409, 907

Popescu, C. C., Misiriotis, A., Kylafis, N. D., Tuffs, R. J., Fischera, J. 2000, A\&A, 362, 138

Popescu, C. C., Tuffs, R. J., Kylafis, N. D., Madore, B. F. 2004, A\&A, 414, 45

Xilouris, E. M., Byun, Y. I., Kylafis, N. D., Paleologou, E. V., Papamastorakis, J. 1999, A\&A, 344,868

Xilouris, E. M., Alton, P. B., Davies, J. I.; Kylafis, N. D., Papamastorakis, J., Trewhella, M. 1998, A\&A, 331, 894 